\title{
Artificial Intelligence in Medical Imaging and its Impact on the Rare Disease Community: Threats, Challenges and Opportunities
}

Navid Hasani, BS ${ }^{a, b}$, Faraz Farhadi, BS ${ }^{a}$,

Michael A. Morris, MD, MS, DABR, DABNM ${ }^{a, c}$, Moozhan Nikpanah, MDª, Arman Rahmim, PhD, DABSNM ${ }^{d, e}$, Yanji Xu, PhD ${ }^{f}$, Anne Pariser, MD ${ }^{f}$, Michael T. Collins, $\mathrm{MD}^{\mathrm{g}}$, Ronald M. Summers, MD, PhD ${ }^{\mathrm{a}}$, Elizabeth Jones, MD, MPH, MBA ${ }^{a}$, Eliot Siegel, MD, FSIIM, FACR ${ }^{h}$, Babak Saboury, MD, MPH, DABR, DABNM ${ }^{a, c, 1, *}$

\section{KEY WORDS}

- Artificial intelligence $\bullet$ Rare diseases $\bullet$ Positron emission tomography $\bullet$ Medical imaging

\section{KEY POINTS}

- Individually Rare, Together Common: Almost 1 in 10 individuals can suffer from one of many rare diseases (RDs).

- The RD community must be recognized and have a seat at the table of Al stakeholders.

- Physicians are expected to be able to diagnose and treat those with common and rare diseases. A similar expectation must be present for Al solutions.

- There is a significant need to recognize the challenge of data imbalance in RDs and to make concerted efforts to provide technical recommendations and guidelines.

- A nationwide RD data repository is an indispensable prerequisite for a fair $\mathrm{Al}$ ecosystem.

- The RD community should be protected from any misuses or abuses of Al systems that lead to discrimination.

\footnotetext{
a Department of Radiology and Imaging Sciences, Clinical Center, National Institutes of Health, 9000 Rockville Pike, Building 10, Room 1C455, Bethesda, MD 20892, USA; ${ }^{\text {b }}$ University of Queensland Faculty of Medicine, Ochsner Clinical School, New Orleans, LA 70121, USA; ${ }^{\mathrm{C}}$ Department of Computer Science and Electrical Engineering, University of Maryland-Baltimore Country, Baltimore, MD, USA; ${ }^{d}$ Department of Radiology, BC Cancer Research Institute, University of British Columbia, 675 West 10th Avenue, Vancouver, British Columbia, V5Z 1L3, Canada; ${ }^{e}$ Department of Physics, BC cancer Research Institute, University of British Columbia, Vancouver, British Columbia, Canada; ${ }^{f}$ Office of Rare Diseases Research, National Center for Advancing Translational Sciences, National Institutes of Health (NIH), Bethesda, MD 20892, USA; ${ }^{9}$ Skeletal Disorders and Mineral Homeostasis Section, National Institute of Dental and Craniofacial Research, National Institutes of Health (NIH), Bethesda, MD, USA; ${ }^{h}$ Department of Radiology and Nuclear Medicine, University of Maryland Medical Center, 655 W. Baltimore Street, Baltimore, MD 21201, USA; ${ }^{i}$ Department of Radiology, Hospital of the University of Pennsylvania, Philadelphia, PA, USA

* Corresponding author. Department of Radiology and Imaging Sciences, Clinical Center, National Institutes of Health, 9000 Rockville Pike, Building 10, Room 1C455, Bethesda, MD 20892, USA

E-mail address: babak.saboury@nih.gov
} 


\section{INTRODUCTION}

Medicine is the art of compassionate care using scientific wisdom and the best available tools. For a physician, each patient is a unique human being, with particular past history and present manifestation. No patient is a "rare patient" or a "common patient"; each patient is a unique human being.

However, modern nosologic taxonomy makes it inevitable to categorize the constellation of clinical findings into specific diseases or syndromes (phenotyping). Here, we group multiple individuals into a specific category. This is a helpful approach because the understanding of the molecular basis of these phenotypic categories enables suitable treatment discovery and implementation. Most of the pharmaceutical treatments are, by design, appropriate for a "group of subjects with a common denominator (specific molecular pathogenesis)".

The cognitive task for clinical medicine includes attentive observation and careful phenotyping of the patients based on their clinical, physical, biochemical, imaging, histopathological, and genetic findings altogether. The result of this process is the appropriate diagnosis. Here is the core of inherent cognitive dissonance: moving between the "unique human-being" and "grouped disease categories"; separation and connectedness at the same time. This paradoxic situation, if unaddressed, manifests itself in a plethora of "diagnostic cognitive biases." 1

We want each patient to be considered unique and individual and at the same time as connected and included in the appropriate categories as possible. Recognition of a group of diseases as "rare diseases" is an attempt to facilitate the "inclusion" into an appropriate category when it is needed (facilitate the connectedness). Acknowledgment of individual differences and advocacy for "personalized medicine" is an attempt to enable individuation (facilitate uniqueness). These one attempts, albeit seemingly contradictory, are two sides of one reality.

Herein, we will discuss how the issue of "inclusion" could be affected by the advancement of artificial intelligence (Al) in medicine. We intend to show that the interaction between rare diseases (RDs) and Al technology is an impactful event: a potential for dreadful consequences as well as colossal progression and improvement. Which path is our path? It is difficult to predict the future but with active engagement, the future can be invented.

Suboptimal performance of Al technologies in patients with uncommon conditions can significantly hinder the clinical utility and translation of Al medical devices into routine clinical practice. ${ }^{2}$ Hence to appropriately incorporate $\mathrm{Al}$ into clinical practice, it is an ethical obligation to address opportunities and concerns related to the diagnosis of RD with Al-based molecular imaging. The societal, economic, and health implications of medical Al for patients with RDs can be 2-fold: (1) through the marginalization and exclusion of those with RDs (discrimination by omission) and (2) through blatant or subtle unregulated postdeployment discrimination or malicious uses of Al technologies (discrimination by commission). This paper aims to explore the medical as well as ethical and societal aspects of using RDs-aware $\mathrm{Al}$ in the health care system.

\section{Prevalence and Disease Burden}

The Orphan Drug Act of $1983^{3}$ classifies a condition as "rare" if it affects fewer than 200,000 people in the United States (Fig. 1). ${ }^{4}$ RDs are rare when considered individually, yet collectively they are common; they consist of more than 7000 to 10,000 identified conditions ${ }^{5}$ influencing more than 350 million people worldwide. ${ }^{6}$ The burden and urgency of the problem have also been investigated recently through the cost-ofillness studies conducted by Everylife FDN ${ }^{7}$ and Navarrette-Opazoand colleagues ${ }^{8}$ which demonstrate large medical and societal expenses as a proxy for unmet medical demands and severity of illness in patients with RDs. RDs encompass a diverse spectrum of conditions, with more than $85 \%$ being single-gene disorders and the remainder being rare malignancies, infections, exposures, and polygenic disorders. ${ }^{9}$ PET imaging has shown an important role in the diagnosis and management of several RDs including multiple endocrine neoplasias, ${ }^{10}$ Von Hippel-Lindau disease, ${ }^{11}$ pheochromocytoma and paraganglioma (PPGL), ${ }^{12}$ familial carcinoid syndrome, ${ }^{13}$ Erdheim-Chester Disease, ${ }^{14}$ and Duchenne muscular dystrophy (DMD). ${ }^{15}$

\section{Enigma of Diagnosis}

Individuals suffering from RDs often undergo numerous consultations, imaging studies, workups, and are frequently misdiagnosed before the final diagnosis is made ${ }^{16}$ - diagnostic odyssey. Additionally, this community suffers from sparse and dispersed medical knowledge, resulting in even fewer effective and approved treatment choices.

Physician's familiarity: Maintaining up-to-date knowledge of particular diagnostic criteria for 7000 various $R D$ s poses a unique challenge for many physicians and specialists. The term "diagnostic odyssey" refers to the time of initial disease symptoms until the final diagnosis of RD, encompassing 


\section{RARE DISEASES}

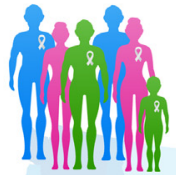

Rare diseases are conditions that impact 200,000 or fewer individuals in the United States

\section{RARE INDIVIDUALLY BUT COLLECTIVELY COMMON}

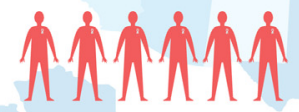

collectively an estimated 25 to 30 million Americans are affected.

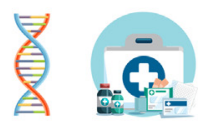

Of the 7,000 identified rare and neglected diseases for which we know the molecular cause, only about 500 have approved treatments.

\section{PREVALENCE OF FEW RARE DISEASES}

『 Hereditary Pheochromocytoma (PCCs) $5 / 100,000$

『 Hereditary-paraganglioma (PGL) $10 / 100,000$

『 MEN2A and MEN2B $<1 / 100,000$

『 Familial carcinoid syndrome $1 / 100,000$ 6-7 years

Fig. 1. Individually Rare, Together Common: Statistics related to the epidemiology of RDs and the importance of early diagnosis.

multiple physicians' consults and diagnostic tests. ${ }^{17}$ The average living person with $R D$ endures a 6 to 7 year long diagnostic odyssey in the US. ${ }^{18}$ According to a Belgian survey, the average level of physician awareness regarding RDs is suboptimal. ${ }^{19}$ Reportedly, most physicians have the desire to diagnose and treat RDs; however, $40 \%$ of general practitioners and $24 \%$ of specialists experience a lack of sufficient time to pursue workups necessary to diagnose RD in clinical practice. ${ }^{20}$ The situation is made more complicated by the fact that only a small number of clinicians with prior exposure are capable of confirming these rare disorders.

Heterogenous presentation of rare diseases: Another related concern is the heterogeneity of symptoms of each disease and sporadic presentation in the body. These factors lead to underdiagnosis or delayed diagnosis of RD. Even when the patient is suspected of having a RD, there is still a significant chance of misdiagnosis due to overlapping symptoms of many RDs. ${ }^{21}$ As a result of these issues, the time-to-diagnosis (TTD) of RDs is significantly prolonged.

A hypothetical solution for this problem, according to Hirsch and colleagues, ${ }^{22}$ is a symptom checker mobile application that assesses patient symptoms to compile lists of likely diseases and subsequently gives safe next step advice. An application can provide a list of specialists, genetic testing, remote self-testing, and continuous telemonitoring of health status, all of which could help lower the TTD of RDs and lower disease burden from this community. ${ }^{22}$

\section{Role of Artificial Intelligence and Medical Imaging}

Recent advancements in information technology, particularly machine learning (ML), and $\mathrm{Al}$ have shown significant prospects of $\mathrm{Al}$-based medical devices (AIMDs) in diagnosis, management, and pharmaceutical development for various diseases. ${ }^{23,24}$ Although these advancements have been less prominent in RDs, AIMDs have demonstrated notable results in several dimensions of common diseases (CDs) diagnosis and prognosis (eg, breast cancers, brain tumors, fractures). ${ }^{25-27}$

One reason for such lack of progress in RDs is the scarcity or heterogeneity of available data on 
a particular RD. As the success of an Al algorithm is directly correlated with the quality and size of datasets, ${ }^{28}$ it is imperative to address these concerns as they pertain to different types of available $\mathrm{RD}$ data.

Although text-mining methods may be promising in the near future, the variability in the quality of data, the heterogeneous nature of Electronic Medical Records (EMR), and the incomplete reporting of information are challenging to semantic interoperability. ${ }^{29,30}$ Imaging data, however, are often standardized, more consistent, and allow the visualization of multiple systems from multiple points of view. We see enormous potential in the imaging data (ie, MR, CT, PET, SPECT) in the training of $\mathrm{Al}$ algorithms as images are typically procured in large quantities and processed in a relatively standardized way, with less inter- or intra-observer variability, therefore providing a great data source for Al training. ${ }^{24}$ Specifically, multivariate imaging data (Total Body PET (TBPET)) that are often standardized can visualize multiple organ systems, which are of particular importance for RD diagnosis, and can lend itself particularly well to use in Al training.

\section{Stigma of Diagnosis}

Although RD-aware Software-as-a-Medical Device (SaMD) is the first essential step toward better integration of $\mathrm{Al}$ in Medicine, our responsibility does not end there and should include the prevention of potential abuses. One must be cognizant of the potential downstream effects and ethical implications of AIMD postdeployment. AIMDs enable continuous and early disease monitoring and detection; while clinically beneficial, this information can be intentionally or unintentionally misused in the context of overgeneralizations by health systems, insurance, or even stigmatization against an already vulnerable population.

Similar concerns were raised with the advent of next-generation sequencing (NGS), involving large-scale whole-exome sequencing (WES) and whole-genome sequencing (WGS), which enabled the third-party gathering of large amounts of patient data. ${ }^{31}$ Sensitive medical information, when made available to a third party without proper deidentification, could potentially have negative repercussions for a person in terms of future insurance, employment, or health care opportunities. As an example, patient WGS findings have been used by heart and lung transplantation teams to decide whether to list an individual as an organ recipient. ${ }^{32}$ Yet, despite the recommendation of the Americans with Disabilities Act (ADA) $)^{33}$ and Genetic
Information Nondiscrimination Act (GINA) ${ }^{34}$ reportedly, many transplant programs across the US continue to be biased and discriminate against those with intellectual and genetic diseases. ${ }^{35}$

Therefore, the issue of data protection and ownership becomes more crucial than ever. It is not far-fetched to assume that many families and patients would have a similar response to $\mathrm{Al}$ based diagnosis or management if they think the results and findings would be used to stigmatize or ostracize them. This foreseeable consequence and potential stigmatization of $\mathrm{Al}$ should be disclosed by the team before the testing is conducted. In the case of genetic testing, many families asserted that had they known the results of WGS to potentially jeopardize the transplantation option, they would have never given consent to testing. This argument further supports the notion that the information regarding third-party use of patient data must be a part of the informed consent form. Publicly available informed consent forms can provide substantial benefit and legal protection in court if information is used for discrimination.

\section{RARE DISEASES \& ARTIFICIAL INTELLIGENCE OPPORTUNITIES AND CHALLENGES}

In this section, we initially explore the bioethics of $\mathrm{RDs}$ in $\mathrm{Al}$, and then discuss the reasons why the medical Al industry and the RD community should actively collaborate in this new era of $\mathrm{Al}$ in medicine. We begin by outlining the reasons why the Al community should create medical devices that are $\mathrm{RD}$-aware which can diagnose $\mathrm{RD}$ with the same accuracy as CDs.

\section{Ethical Considerations Related to Artificial Intelligence-Based Diagnosis and Management of Rare Diseases}

a. Exclusion: The scarcity of diverse representation in precision medicine and other biomedical research has been a well-known problem. ${ }^{36}$ Ethical $\mathrm{Al}$ is diverse in its training data and performance outcomes. ${ }^{37}$ Lack of inclusion of RDs in AIMDs does not align with bioethical values of beneficence and justice. For instance, rare genetic variants and uncommon conditions may be overlooked or their association with common, complex diseases can be misinterpreted. ${ }^{38}$ The exclusion of patients with RDs at the training stage of the Al system subsequently leads to worse performance due to sampling bias and the absence of generalizability. Additionally, this inclination can create bias in future medical studies conducted based on $\mathrm{Al}$-produced data. To maximize clinical 
benefits and minimize harm, the SaMD must perform with safety (to prevent injuries and hazards), efficiency (able to effectively solve the problem at a reasonable cost), and equity (that the advantages of the application are shared fairly by all). ${ }^{39}$ Thus, the deployment of Al that excludes a subgroup of individuals would diverge from any progress toward the goal of maximizing clinical benefits and addressing health inequities in historically neglected populations.

b. Stigmatization: Personal health information (PHI) handled and enhanced by AIMDs must not be used against the patient for insurance, employment, delaying, or denying of medical care. ${ }^{40}$ For example, there are differences across transplant programs when it comes to the availability of standards and listing policies for genetic diseases and intellectual disabilities. ${ }^{35}$ Confidentiality transgressions with unethical usage of $\mathrm{PHI}$ may occur endangering the social fabric of this already disadvantaged community of patients with RDs.

c. Consent for revealing incidental findings by rare diseases-aware artificial intelligence: Al can open the door for vast amounts of patient data and sensitive information to play a role in clinical decision making. ${ }^{41}$ The chance of incidental findings is inherent in medical imaging studies. Using Al-based detection algorithms can further enhance rates of incidental findings in these studies. The discovery of early signs of RDs could be among incidental findings detected in imaging studies with the assistance of RD-aware detection algorithms. For example, an automatic Al-based wholebody PET/CT ordered for the staging of a tumor can detect a new disease that was not expected at the time of testing. Are we obligated to reveal the new information to the patient? If not, how much information should the clinician reveal to the patient? Revealing the data we did not intend to find to patients has ethical and psychological implications that need to be addressed. Clear communication regarding the use of $\mathrm{Al}$ findings is necessary for all patients including those with RDs. This is because the health provider and patients' knowledge of Al capabilities and expectations of use of Al findings may often be different. Regardless of the fact that there is significant discussion regarding what findings should be returned, patients will have vastly diverse experiences based on which physicians and health care professionals they received care from. The same issues were present for NGS. ${ }^{42}$ We must learn from the implementation of NGS in clinical practice for the successful translation of $\mathrm{Al}$ into precision medicine and RD diagnosis and management.

\section{Why Should the Artificial Intelligence Community Recognize Rare Diseases as One of the Stakeholders?}

To achieve the full potential of these technologies and create a sustainable Al ecosystem, Al medical devices should be integrated into an ethical platform that patients and physicians can trust [Navid Hasani and colleagues article, Trustworthy Artificial Intelligence in Medical Imaging, in this issue]. ${ }^{43,44}$ There are 5 reasons why the Al industry in health care needs to consider the inclusion of RDs:

(1) The Food and Drug Administration (FDA) and the scientific community are aware that the performance of an Al medical device depends on the training data. It is not far-fetched that the effectiveness of an Al trained and evaluated on common conditions will be incapable of use in RDs. Thus, by neglecting RDs at the Al training and development stage, the developer is unable to claim any application of the algorithm for the diagnosis or management of those with RDs.

(2) The RD community is vulnerable and has been historically neglected by researchers, drug developers, medical specialists, and policymakers. ${ }^{45}$ There are substantial disease and financial burdens placed on surviving patients diagnosed with an uncommon disease and their families. ${ }^{46}$ Therefore, in the era of $\mathrm{Al}$ in medicine, it is imperative to use advancements of Al-based medical imaging to appropriately diagnose and manage patients with RDs, minimizing the magnitude of potential harm to this underserved population. ${ }^{47}$

(3) If RDs are neglected and Al is deployed into health care, the potential absence of accurate and consistent RD-related diagnosis may worsen an already devastating situation. This would have significant negative implications for both the RD and the Al community which hinders the society's and medical community's trust in Al. When patients refer to their physician for certain symptoms, they expect an accurate diagnosis. If the physician works with an Al-based medical imaging device system that can assist in the diagnosis of diseases, that device must be accurate in detecting RDs as similarly expected for CDs. Otherwise, when Al encounters an uncommon disorder phenotype it may depict aberrant behavior, categorize the phenotype into a 
CD group, or label it as unknown. Diversity in data includes the diversity of ethnic groups, genders, ages, and diseases. In terms of disease diversity in training data, there must be an assorted range of rare and common illnesses that the AIMDs can detect. ${ }^{48}$ Inability to do so due to an underrepresentation of uncommon conditions can conceivably induce biases in the performance and evaluation of disease burden. ${ }^{49} \mathrm{Al}$ developers must be aware of such biases to avoid potential Alinduced discrimination further widening the health disparity gap. ${ }^{50}$

(4) As sustainable growth of Al in society needs the definition of trustworthiness, it is ethical and obligatory to consider RDs. The advent of $\mathrm{Al}$ tools and their associated abilities have created a new ethical and social obligation for intelligent interventions that, if leveraged appropriately, can significantly improve RDrelated diagnosis, treatment, and patient support systems. Suboptimal performance of an Al diagnostic tool that disproportionately impacts the RD community would substantially limit translation to clinical practice as well as trustworthiness of AIMDs in health care. ${ }^{22}$

(5) The inclusion of RDs and data diversity Improves the robustness of $\mathrm{Al}$ systems and their utility in a variety of clinical settings. Enhanced performance of such systems contributes to a more diverse market, hence more profitable for Al developers. Therefore, the financial and social advantages of accurate and successful Al medical devices would be beneficial for the Al community and developers. Furthermore, studying and gathering data on RDs can provide insights into the intricacies of biological mechanisms underlying many CDs which can be leveraged by the developers to further improve the performance and robustness of future AIMDs.

\section{Why Should the Rare Disease Community Want to Be an Active Stakeholder in the New Era of Artificial Intelligence in Medicine?}

Now, let us examine this from the perspective of the RD community and patient advocacy groups. Patients with RDs confront a number of significant challenges: late diagnosis and misdiagnosis, limited disease-modifying therapies, proper response to therapies, valid monitoring tools as well as little private-sector $R \& D$ and public health prioritization due to a low number of diagnosed patients in each disease category. ${ }^{51-53}$ The power of Al can be used to address these obstacles. As such, the implications of $\mathrm{Al}$ in health care are critical for RD diagnosis and management. This period can be the best or the worst of times for RDs diagnosis and management.

There are great opportunities for the inclusion of $\mathrm{RD}$ data into Al-based diagnostic tools, especially considering the growing complexity of medical data (Big Data) ${ }^{54}$ and advancements in deep learning. Al tools can analyze and learn from large and complex datasets for quantitative assessment purposes ${ }^{55}$ such as using PET for objective detection and characterization of neuroendocrine tumors. ${ }^{56} \mathrm{Al}$-based decision support systems may be developed to assist clinicians in their differential diagnosis of RD. ${ }^{21}$ Other algorithms can assist in therapeutic research and development (i.e. human-on-a-chip or clinical-trial-on-a-chip). ${ }^{57,58}$ Recent Al research in meta-learning has demonstrated unprecedented success in training $\mathrm{Al}$ with limited data (to mimic the scenario for RD) using discriminative ensemble learning, fast few-shot transfer learning, and generalized zero shot learning (ZSL) for chest X-ray diagnosis. ${ }^{59-61}$ Table 1 provides a short list of examples of how $\mathrm{ML}$ and $\mathrm{Al}$ algorithms are currently being used to further advance RD screening, treatment, and research.

At the same time, most efforts in the era of $\mathrm{Al}$ in medicine are focused on advancing CD diagnosis and management, neglecting the inclusion of RDs. This is an essential detail because, at a time whereby AIMDs are progressively developed and incorporated into clinical practice, we must highlight the maleficence of using AIMDs not capable of detecting RDs as accurately as CDs. As such, the deployment of $\mathrm{Al}$ tools into clinical practice could bear detrimental impacts on patients with undiagnosed RDs. Considering the automation bias associated with $\mathrm{Al}$ in health care, reliance on Al systems incompatible with RDs may promote misdiagnosis or prolong the TTD for undiagnosed patients with RDs. ${ }^{70}$ A prolonged TTD not only exacerbates suffering but also can lead to further financial and health debilitation of patients and families. ${ }^{16}$

\section{HOW TO ENABLE THE DEVELOPMENT AND IMPLEMENTATION OF RARE DISEASES- AWARE ARTIFICIAL INTELLIGENCE SOLUTIONS \\ Outreach and Collaboration with all Stakeholders}

In a sustainable Al ecosystem, active collaboration exists between all stakeholders throughout the lifecycle of AIMDs. The recognition and inclusion of RD patient advocacy groups (PAGs) in the circle of stakeholders are important and must be 
Table 1

Tangible examples of Al improving RD diagnosis and management

Title

Symptoms checker website

RD diagnosis support using facial features

Al for RD drug repurposing

Al-based genetic devices

Al-based Human-on-a-chip and Clinical-Trial ona-Chip drug development

Al and PET Enables Orphan Drug Development

\section{Description}

Al-based applications allow patients or physicians to input presenting symptoms to determine the probabilistic differential diagnosis based on various RD databases. ${ }^{62}$

Gurovich and colleagues ${ }^{63}$ proposed a novel facial recognition algorithm called DeepGestalt, powered by FDNA inc., that classifies distinct facial features from photos of individuals with rare neurodevelopmental and congenital disorders. The phenotypic analysis algorithm is trained on 100,000 images and can distinguish between 200 different rare syndromes achieving $91 \%$ accuracy in identifying the correct diagnosis in the top 10 suggestions. ${ }^{63}$

Lee and colleagues ${ }^{121}$ used the URSAHD (unveiling RNA sample annotation for human diseases) machine learning framework to apply genetic and molecular information from hundreds of complicated conditions to medication repurposing. Similarly, Ekins and colleagues created an end-to-end ML algorithm that leverages ${ }^{64}$ large quantities of screening data to predict bioactivities for therapeutic targets and molecular properties with increased levels of accuracy.

The ML algorithm "Xrare" proposed by Li and colleagues $^{65}$ jointly uses phenotypic and genetic evidence for the identification of causative gene variants in RDs and the prioritization of these diagnoses.

Almost $95 \%$ of RDs do not have an approved treatment option ${ }^{66}$. Recent Al systems have shown great promise in promoting drug development or repurposing of available therapeutics. For example, the human-on-a-chip (HoaC) or organ-on-a-chip ${ }^{58,67}$ is a novel CNNbased model that mimics human organ functionality in interconnected in-vitro bioengineered micro-physiological systems. This medical imaging technology can recapitulate clinical trials on a chip (CToCs) ${ }^{68}$ lowering the costs and recruitment barriers related to conducting RD clinical trials (scarce number of RD patients) ${ }^{57}$. This technology promotes research and development of novel PET radioactive tracers for tumor detection and treatment by introducing human phenotypic models early in the drug discovery process.

Al-based PET kinetic modelings have the potential to yield valuable data regarding the pharmacokinetics of investigational pharmaceuticals with a small number of participants. 
Table 1

(continued)

Title Description

Radiophenomics: Al-based medical imaging for recognition of rare disease manifestation

TB-PET powered with Al capabilities has enormous potential to identify imaging patterns suggestive of rare diseases phenotypes. Therefore, using image-based phenotyping (radiophenomics) the $\mathrm{Al}$ can suggest a possibility of a rare disease pattern to the physician.

Al-based medical imaging management of rare diseases

Pretest probability is the likelihood of having a condition before the results of a diagnostic test are known ${ }^{69}$. Al algorithms can be trained to understand the pre-test probability and incorporate it into screening and management in individuals with rare diseases. The accuracy of the probability scale generated by $\mathrm{Al}$ and the ideal threshold used on the image may vary depending on the pre-test probability.

enforced by appropriate regulatory committees. Stakeholders involved and affected (directly or indirectly) throughout the lifecycle of $\mathrm{Al}$ include patients, physicians, and all the relevant providers, health care systems, payors, regulatory agencies, professional societies, and patient groups. Inclusive, transparent, and active engagement of these stakeholders throughout the development, design, and postdeployment surveillance of Al systems is needed. Patient advocacy groups specific to each RD are trusted by patients and physicians and can provide substantial assistance in building trust in using Al to diagnose RDs. By active engagement, RD patient leaders and PAGs can communicate concerns related to denying or delaying health care services through discussions with stakeholders and regulatory agencies.

There must be clear and continuous outreach efforts to educate patients regarding Al advancements in RD diagnosis and treatments. ${ }^{71}$ Grass-root organizations should educate the RD community and patients on the challenges and opportunities of Al-based RD diagnostic tools while building trust and helping to identify inappropriate adoption of $\mathrm{Al}$ in health care. Patient advocacy groups can be used as a liaison between the needs of patients with RDs, physicians, and the Al development industry.

To further advance the utility of Al tools in health care, continuous medical education is necessary. Such efforts directed toward medical professionals can facilitate the timely diagnosis and treatment of patients with RDs in outpatient and inpatient settings. ${ }^{72}$ Clinicians should be able to maintain a baseline knowledge of RDs and keep up to date with new treatments and diagnostic tools. PAGs and organizations can convey the current state of art Al-based RD diagnosis to physicians and request that knowledge is transferred from physicians to the patients.

The National Organization of Rare Diseases (NORD), Genetic Alliance (GA), the Chan Zuckerberg Initiative ${ }^{73}$ are instrumental in increasing the capacity of RD patient groups, issuing policies, and incentivizing industry for more efforts toward diagnosis and treatment of RDs. ${ }^{72,74}$ NORD now consists of more than 2000 and the GA more than 600 patient advocacy organizations. ${ }^{75,76}$ In addition to advocating for $\mathrm{RD}$, these organizations can also collaborate with developers and physicians to raise awareness for Al-based diagnosis and strategies to apply Al powers to use the limited amount of available data to better treat RDs.

\section{Rare Diseases Data Meta-Repositories}

The ability of Al technologies to integrate and analyze data from different structured and unstructured sources (eg, radiomics, patient registries, anecdotal evidence, genetic variation, and molecular profiling) $)^{77,78}$ can be used to overcome $\mathrm{RD}$ data challenges (eg, low diagnostic rates, limited number of patients in each RD category, geographic dispersion, and heterogenous clinical presentation). Nevertheless, there is a lack of central $\mathrm{RD}$ data repositories for $\mathrm{Al}$ training. ${ }^{23}$ Although 
there are specialized repositories in existence, such as The Cancer Imaging Archive (TCIA), ${ }^{79}$ it is vital to further expand the availability of similar datasets for RD and link different types of data together for each patient.

Sharing of anonymized health data sets enables a deeper and broader understanding of the nature of $\mathrm{RD}$ and affected patient populations. ${ }^{80}$ The move toward "Big Data" provides substantial evidence for advancing model training and tuning, shortening the TTD, clinical outcomes, as well as the development of treatments for RDs. ${ }^{81}$ Patients are often supportive of safe and transparent sharing of their anonymized electronic health records (EHR) for research. ${ }^{82}$ Consenting to $\mathrm{PHI}$ sharing, as Figueiredo states, may also be a method of repaying society's investment in science through public-funded research or charity. ${ }^{83}$ To this end, it is a social responsibility of public institutions, private centers, schools, hospitals, etc. to share their high-quality, exquisitely curated, privacy-preserved diverse health and consumer data on patients with RDs for curating a central $\mathrm{RD}$ and $\mathrm{CD}$ data repository for Al training and testing. Professional societies (e.g. Society of Nuclear Medicine and Molecular Imaging (SNMMI), Society of Imaging Informatics in Medicine (SIIM), Radiological Society of North America (RSNA), and American Medical Informatics Association (AMIA), among others) can help address institutional barriers to data sharing and act as a liaison between stakeholders and associated organizations.

The National Institutes of Health $(\mathrm{NIH})$ are also committed to providing long and short-term support to the RD community and to promoting $\mathrm{Al}$ research in areas whereby there may otherwise be insufficient attention or action. ${ }^{84}$ To achieve this goal, NORD and the Rare Disease Clinical Research Network (RDCRN ${ }^{85}$ at the National Center for Advancing Translational Sciences (NCATS) have curated $\mathrm{RD}$ registries that are available for patients, researchers, and clinicians. ${ }^{86}$ Such registries not only facilitate communication between health care professionals, patients, and the multidisciplinary team but also highlight critical aspects of data quality, governance, and access. ${ }^{77}$

\section{Rare Diseases Awareness at Artificial Intelligence Conceptualization/Development Stage}

It is of utmost importance for RDs to be considered in all stages of model and decision support system development. Specifically, RD awareness at the design stage relates to the inclusion of RDs alongside CDs in datasets used for model training, validation, and testing of performance. The model should be able to categorize each disease pattern into a certain identified rare or $C D$ pattern. For example, the Al-based imaging device should be able to perform a classification task related to $R D$ and $C D$ identification with reasonable accuracy and precision. For an Al-based imaging device, $\mathrm{RD}$ categories must be included in image recognition, annotation of radiomics, disease ranking, diagnosis, and prediction. To accomplish this, the diversity of RD and CD patterns must be included in the derivation and validation of $\mathrm{Al}$ models. This conceptualization minimizes chances that a rare event or incidence triggers either an aberrant error, is misplaced into a CD category based on resemblance, or is identified as an unknown pattern.

\section{Class imbalance}

Inductive approaches are employed in computational phenotyping algorithms to find correlations or patterns within datasets. The diagnostic accuracy and informative value of the resulting phenotyping tools are determined by the amount, variety, and accuracy of data used in model training and testing. ${ }^{87}$ As a result, creating a representative database for algorithm training is critical to the effectiveness of computational phenotyping in RDs. ${ }^{88}$ However, inherent to RDs, available sample sizes for the training of algorithms are often limited. This leads to class imbalance issues. Through multi-institutional collaborations (direct sharing of data and/or use of federated learning), physicians and scientists should make efforts to address this limitation. In any case, even with much needed concerted efforts, class imbalance problems are likely to persist.

To tackle this, first, we note that poor performances in class imbalance problems can be hidden if limited metrics are used for assessment; for example, for an Al algorithm that performs poorly at detecting a rare minority class, overall area-under-the-receiver-operator-characteristiccurve (AUC ROC), accuracy or specificity used as metrics may still show excellent performances ("the accuracy paradox"), due to the dominance of the majority class (e.g. healthy patients or $C D$ ). As such, a range of evaluation metrics especially those de-emphasizing true negatives (i.e. correctly classified majority class) should be reported such as recall, precision, F1 score, false-discovery rate, and the area-under-the-precision-recallcurve (AUC PR) $)^{89}$ to better reveal poor performances of $\mathrm{Al}$ algorithms in class imbalanced datasets.

To tackle poor performances in classimbalanced problems, computational methods 
including majority class undersampling and/or minority class oversampling/data augmentation can be invoked (e.g. SMOTE, ${ }^{90}$ ADASYN $^{91}$ ). In particular, data augmentation can be performed on the training data to increase the size of datasets and limit overfitting. Conventional techniques of image transformations can be used such as rotating, cropping, and zooming. ${ }^{92}$ Additionally, Al itself might be capable of providing solutions to this matter; for example, using Generative Adversarial Networks (GANs) as used in many disciplines to generate new, synthetic instances of data that can pass for real data ${ }^{93}$ and used for data augmentation purposes. ${ }^{94}$ Use of Al-based Style Transfer $^{95}$ for data augmentation can also be further explored.

Instead of augmenting/manipulating the datasets directly, it is also possible to use other techniques that enhance the overall model architecture, making it more suited for a classimbalanced problem. Some of these include using weighted loss functions such as the focal loss $^{96}$ that gives different weights for calculating the loss values related to the majority and the minority classes or using penalized models such as cost-sensitive support vector machine $(\mathrm{SVM})^{97}$ with inbuilt hyperparameters that can be weighted as per the importance of each class (with the minority class getting more weight). Certain other techniques such as anomaly detection ${ }^{98}$ attempt to solve this issue by redefining the classimbalance problem (i.e. with a majority and a minority class) into a problem of outlier/novelty detection (with the minority class being the outlier/novelty) in a normal (majority class) dataset.

To this end, while training an Al algorithm, the prevalence and/or importance of RD can be further enhanced (as discussed above), enabling the derivation of Al models that are effective in detecting common conditions as well as uncommon diseases. This approach is similar to the expectation that exists in medical education, including board certification examinations, to train physicians that are capable of diagnosing and treating patients with CDs as well as RDs. Therefore, it is critical to recognize this challenge and to make active efforts to provide technical best practice recommendations and guidelines.

\section{Oversight and Regulation}

Appropriate development and deployment of Albased medical tools necessitate continuous ethics, safety, and performance assessment by objective oversight and evaluation frameworks that are inclusive of RDs. At this exciting transitional stage, whereby $\mathrm{Al}$ is increasingly used in health care across the world, there are numerous opportunities compounded with Al governance difficulties. ${ }^{99}$ The federal and local policies are often not able to keep up with the exponential growth of Al technology in health care. For this reason, a regulatory agency or public organization can ensure the proper implementation of Al-aware policies for RD diagnosis and treatment. The creation of legislation may potentially follow an industrial and political complex path ${ }^{100}$ and must address the collective needs of patients with RDs. In regard to the US, the regulation of drugs, biologics, and devices are predominantly overseen by the FDA. ${ }^{44}$

One aspect of regulation for RD-aware $\mathrm{Al}$ entails inclusivity monitoring, which can be conducted either by the FDA or an independent professional credentialing organization similar to the certification and recertification of medical professionals. The regulatory body should ensure the generalizability of the Al to all patients suffering from a particular condition regardless of extraneous personal characteristics. Individuals who are underrepresented or suffer from RDs should not be excluded from the creation, assessment, and deployment stages of Al systems. Appropriate validation testing on standardized sets that include a diverse patient population, including rare or unusual presentation of disease, is critical to assess for the presence of bias in results. ${ }^{37,48}$ More specifically, the oversight must include the assessment of performance that is, equitable, diverse, and includes relevant CDs and RDs. To achieve this goal, oversight can enforce and monitor the safety, security, ethics of SaMDs at every stage of conceptualization, development, and deployment. $^{88,99}$

Another highly important regulatory aspect pertains to information security and the nondiscriminatory use of Al for RD diagnosis and management. If Al-based medical imaging systems improve the diagnostic capabilities for RDs, the knowledge of this RD cannot be used by third parties to pose potential health, financial, or societal harm (stigmatization) toward the individual. Thus, the implementation of Al systems in health care will require a recalibration of confidentiality and other core tenets of professional ethics. As discussed previously, gathering more complex medical information on patients with RDs require legal protection against unintended harm (i.e. available informed consent forms). Due to the novelty of $\mathrm{Al}$ in medicine, there have not been many legal cases against Al, however, 
predictably, potential unintended discrimination and stigmatization can happen as the result of widespread Al use in medicine. Patients with RDs are even more prone to discrimination and have been previously neglected care and treatment due to their illnesses or survival rates. Medical programs and health institutions should rethink their strategy, ensuring individualized evaluations and preventing the exclusion of patients based on their RD group membership. ${ }^{35}$ Additionally, Al-based diagnosis of a RD and associated data should not be used to discriminate against this group or deny or delay care. We need to create legal protections for vulnerable populations including those with diagnosed RDs. Acknowledgment and adoption of policies similar to the Genetic Information Nondiscrimination Act (GINA) ${ }^{101}$ that prohibits discrimination by health insurance and employers for information for medical Al may be imperative if we are to prevent harm before the deployment of $\mathrm{Al}$ for the management and diagnosis of RDs.

\section{EMERGING APPLICATIONS OF ARTIFICIAL INTELLIGENCE IN MOLECULAR IMAGING OF RARE DISEASES \\ Synergism of Artificial Intelligence and Positron Emission Tomography to Accelerate Orphan Drug Development}

Less is different

Orphan drug development is not merely drug development on a smaller scale. The challenges are not just the limited market. ${ }^{102}$ Here, less is different. Considering the limited number of affected individuals eligible for clinical trials, translation of preclinical targets to clinical phase studies requires extra care (precise translation). ${ }^{103}$ Drug development, in general, benefits from Al for more successful target selection ${ }^{104}$ as less than $9 \%$ of investigational drugs entering phase 1 clinical trials reach the FDA approval. ${ }^{105}$ For CDs, this is an improvement but for RDs, it is essential: population-based data for physiologically based pharmacokinetic modeling might be insufficient and the limited number of eligible candidates makes the pharmacokinetic estimations more prone to error due to smaller sample size and sparse data. Al-based PET kinetic modelings have the potential to produce valuable data regarding the pharmacokinetics of the investigational drugs with a limited number of subjects by the implementation of TB-PET dynamic imaging (Figs. 2 and 3). ${ }^{106}$ The depth of such information in addition to Al-augmented pharmacochemistry provides a platform for nuanced structural fine-tuning of the selected target. ${ }^{107}$ In summary, advanced computational techniques in systems biology and multi-scale modeling in addition to the novel technology of dynamic TB-PET imaging and Al-empowered complex systems modeling can aid in the creation of virtual replicates of patients, also known as digital twins. ${ }^{78,108}$
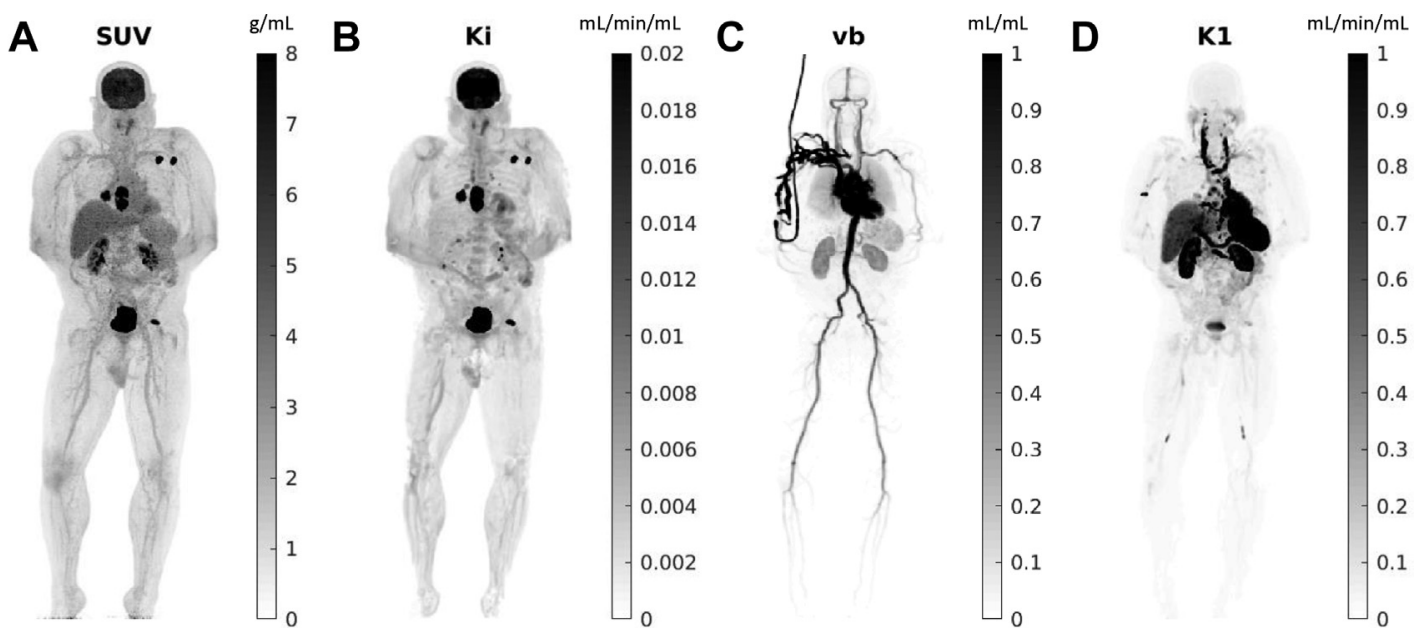

Fig. 2. Total-body parametric images estimated from a 60-min dynamic 18F-FDG scan of a patient with metastatic cancer on the UEXPLORER: $(A)$ SUV, $(B)$ FDG net influx rate Ki, $(C)$ fractional blood volume vb, and $(D)$ FDG delivery rate K1. (From Wang Y, Li E, Cherry SR, Wang G. Total-Body PET Kinetic Modeling and Potential Opportunities Using Deep Learning. PET Clinics. 2021;16(4):613 to 625.: with permission.) 


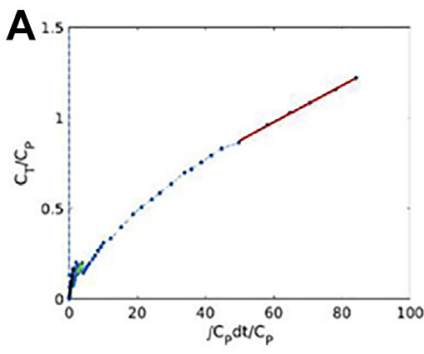

C

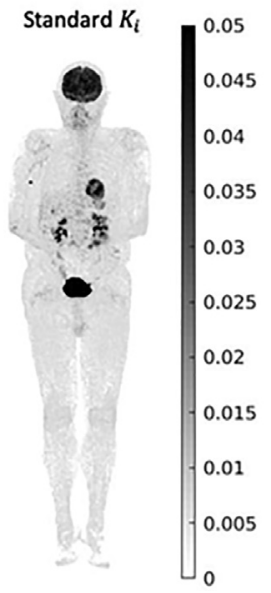

B
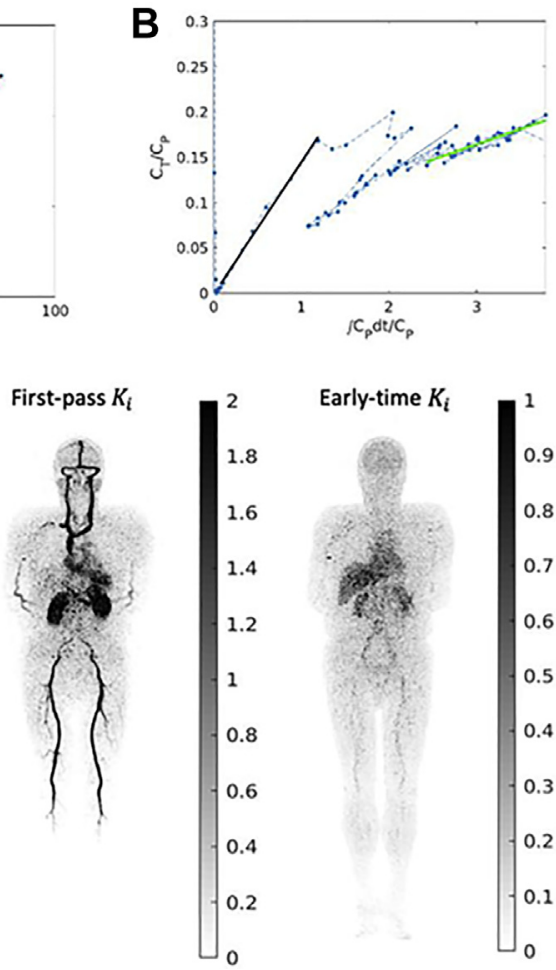

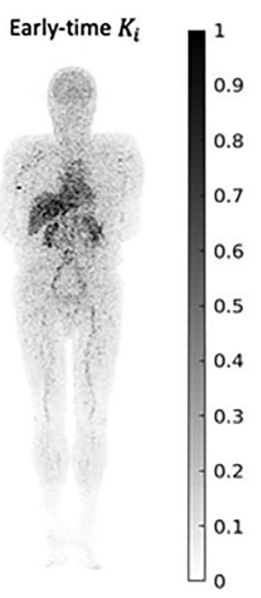

Fig. 3. High-temporal resolution Patlak plot and parametric imaging.30 (A) Patlak plot of 1-h dynamic FDG data; $(B)$ zoom-in of the first 2- $\min$ data; (C) parametric images of the slope at 3 different phases-standard Ki (30-60 minutes), first-pass Ki (20$30 \mathrm{~s})$, and early-time Ki (1-2 minutes). Shown are the MIP images. The unit of $\mathrm{Ki}$ is $\mathrm{mL} / \mathrm{min} / \mathrm{mL}$. (From Wang $\mathrm{Y}, \mathrm{Li}$ E, Cherry SR, Wang G. Total-Body PET Kinetic Modeling and Potential Opportunities Using Deep Learning. PET Clinics. 2021;16(4):613 to 625.: with permission.)

\section{Diagnosis of Rare Diseases with Artificial Intelligence and Molecular Imaging- Can We Shorten the Diagnostic Odyssey?}

The multi-regional nature of routine PET images (from the base of skull and neck, through chest, abdomen, and pelvis to the upper aspect of thighs) provides comprehensive coverage to combine various imaging findings in many organs and assemble a multi-regional multi-organ pathology profile. Such information is invaluable for imagebased phenotyping (radiophenomics). A simple suggestion by the Al software can remind the nuclear medicine physician of a possibility of a RD pattern and this may trigger a further investigation if deemed appropriate based on other clinical findings.

\section{Artificial Intelligence-Based Positron Emission Tomography Solutions for Management of Patients with Rare Diseases}

It is crucial to develop RD-specific Al algorithms that understand the pre-test probability of screening and diagnosis in patients with RDs. For instance, as a medical imaging physician is reviewing an imaging study of a patient with MEN2A syndrome, abnormal adrenal gland findings could be more for an adrenal malignancy. Al algorithms should be developed to use radiomics information to incorporate such clinical information pertinent to the management of patients with RDs. ${ }^{109}$ Both the accuracy of the probability scale (i.e. $70 \%$ chance of MEN2A) and the optimal threshold that is applied to the image may vary based on the pretest probability.

PET is an important imaging modality in management of patients with RDs, ${ }^{110-112}$ such as multiple endocrine neoplasia syndromes (MEN1, MEN2A, MEN2B), ${ }^{113}$ tumoral calcinosis, ${ }^{114}$ VonHippel Lindau (VHL), ${ }^{115}$ familial carcinoid syndrome, ${ }^{116}$ metastatic pheochromocytoma, and paraganglioma, ${ }^{117}$ hemangioendothelioma, and other hereditary cancer syndromes. ${ }^{118}$ Appropriately employing the capabilities of $\mathrm{Al}$ in conjunction with PET could improve monitoring and management of RDs. Al-based PET models have already shown to be capable of performing segmentation, detection, and classification tasks more objectively and efficiently than expert clinicians. ${ }^{119,120,121}$

\section{SUMMARY}

If applied properly, Al-based Positron Emission Tomography (PET) has enormous potential to revolutionize the detection and management of RDs, particularly considering the richness of data in 
emerging TB-PET. ${ }^{122,123}$ However, the RDs community and stakeholders must be recognized and present at the table of Al stakeholders. A national archive of RD data is a necessary precondition for a just Al environment. Al medical systems should provide a clear notice stating that any differential diagnostic support system may miss some RDs, if not appropriately trained and validated. There should be technical guidelines on data augmentation for Al training, including a greater proportion of $\mathrm{RD}$ data in training sets than is currently the case. The RD community should be safeguarded against any exploitation or abuse of Al systems that result in discrimination toward those living with these conditions. This could be accomplished in a variety of ways, including teaching medical/scientific communities' ethical leaders about the critical nature of RD inclusion, as well as through regulatory mandates.

\section{CLINICS CARE POINTS}

- Medicine is the art of compassionate care using scientific wisdom and the best available tools. Al medical devices (AIMDs) are among those tools. It is the responsibility of physicians and healthcare system to understand the beneficial uses and dangerous misuses of this new technology, particularly in relation to the rare disease community.

- If the performance of AIMDs is not evaluated in rare diseases carefully, there might be an ethical obligation to disclose the potential harms (for example, the device could have a clear disclaimer stating that some RDs may be missed in the differential diagnosis support system).

- The rare disease community should be recognized as a stakeholder in the lifecycle of AIMDs and they should be involved in all phases of development, deployment, and oversight.

- Al solutions for RD patients could be actualized through sustainable cooperation among all stakeholders (from creation of data metarepositories, to social awareness at all phases of development and implementation).

\section{ACKNOWLEDGMENTS AND DISCLOSURES}

This research was supported in part by the Intramural Research Program of the NIH, Clinical Center. The opinions expressed in this publication are the author's own and do not reflect the view of the National Institutes of Health, the Department of Health and Human Services, or the United States government.

The authors acknowledge valuable feedback by Shadab Ahamed on the class imbalance problem. The authors acknowledge and thank Dr. David Magnus for his contributions.

\section{REFERENCES}

1. Kwok ESH, Calder LA, Barlow-Krelina E, et al. Implementation of a structured hospital-wide morbidity and mortality rounds model. BMJ Qual Saf 2017;26(6):439-48.

2. Sharma N, Aggarwal LM. Automated medical image segmentation techniques. J Med Phys 2010; 35(1):3-14.

3. Act C. Orphan drug act of 1983. Public L 1983;97: 414. Available at: https://www.fda.gov/media/99546/ download.

4. Office of the Commissioner. Rare diseases at FDA. Available at: https://www.fda.gov/patients/rarediseases-fda. Accessed July 6, 2021.

5. Ekins S. Industrializing rare disease therapy discovery and development. Nat Biotechnol 2017; 35(2):117-8.

6. RARE facts. Available at: https://globalgenes.org/ rare-facts/. Accessed July 6, 2021.

7. Burden of Rare Disease Study - EveryLife Foundation for Rare Diseases. Available at: https:// everylifefoundation.org/burden-study/. Accessed September 18, 2021

8. Navarrete-Opazo AA, Singh M, Tisdale A, et al. Can you hear us now? The impact of health-care utilization by rare disease patients in the United States. Genet Med 2021. https://doi.org/10.1038/ s41436-021-01241-7.

9. FAQs about rare diseases. Available at: https:// rarediseases.info.nih.gov/diseases/pages/31/faqsabout-rare-diseases. Accessed September 18, 2021.

10. Multiple endocrine neoplasia type 1 - NORD (national organization for rare disorders). 2015. Available at: https://rarediseases.org/rare-diseases/multipleendocrine-neoplasia-type-1/. Accessed July 21, 2021.

11. Von Hippel-Lindau Disease - NORD (National Organization for Rare Disorders). 2015. Available at: https://rarediseases.org/rare-diseases/von-hippellindau-disease/. Accessed July 21, 2021.

12. Pheochromocytoma - NORD (National Organization for Rare Disorders). 2015. Available at: https:// rarediseases.org/rare-diseases/pheochromocytoma/ . Accessed July 21, 2021.

13. Carcinoid syndrome - NORD (national organization for rare disorders). 2015. Available at: https:// 
rarediseases.org/rare-diseases/carcinoid-syndrome/ Accessed July 21, 2021.

14. Erdheim Chester Disease - NORD (National Organization for Rare Disorders). 2015. Available at: https://rarediseases.org/rare-diseases/erdheimchester-disease/. Accessed July 22, 2021.

15. Duchenne muscular dystrophy - NORD (national organization for rare disorders). 2015. Available at: https://rarediseases.org/rare-diseases/duchennemuscular-dystrophy/. Accessed July 21, 2021.

16. Black N, Martineau F, Manacorda T. Diagnostic odyssey for rare diseases: exploration of potential indicators. Available at: https://piru.ac.uk/assets/ files/Rare\%20diseases\%20Final\%20report.pdf. Accessed July 7, 2021.

17. Contributer $\mathrm{G}$. The rare disease diagnostic odyssey: a medical student's perspective. Available at: https://www.findacure.org.uk/2018/07/06/the-raredisease-diagnostic-odyssey-a-medical-studentsperspective/. Accessed July 6, 2021.

18. The Lancet Diabetes Endocrinology. Spotlight on rare diseases. Lancet Diabetes Endocrinol 2019; $7(2): 75$.

19. Vandeborne L, van Overbeeke E, Dooms M, et al. Information needs of physicians regarding the diagnosis of rare diseases: a questionnaire-based study in Belgium. Orphanet J Rare Dis 2019;14(1):99.

20. Hempel H. Accurate diagnosis of rare diseases remains difficult despite strong physician interest. 2014. Available at: https://globalgenes.org/2014/ 03/06/accurate-diagnosis-of-rare-diseases-remainsdifficult-despite-strong-physician-interest/. Accessed July 22, 2021.

21. Alves R, Piñol M, Vilaplana J, et al. Computer-assisted initial diagnosis of rare diseases. PeerJ 2016;4: e2211. https://doi.org/10.7717/peerj.2211.

22. Hirsch MC, Ronicke S, Krusche M, et al. Rare diseases 2030: how augmented Al will support diagnosis and treatment of rare diseases in the future. Ann Rheum Dis 2020;79(6):740-3.

23. Brasil S, Pascoal C, Francisco R, et al. Artificial intelligence $(\mathrm{Al})$ in rare diseases: is the future Brighter? Genes 2019;10(12). https://doi.org/10. 3390/genes10120978.

24. Schaefer J, Lehne M, Schepers J, et al. The use of machine learning in rare diseases: a scoping review. Orphanet J Rare Dis 2020;15(1):145.

25. Becker AS, Marcon M, Ghafoor S, et al. Deep learning in mammography: diagnostic accuracy of a multipurpose image analysis software in the detection of breast cancer. Invest Radiol 2017;52(7):434-40.

26. Comelli A, Stefano A, Russo G, et al. A smart and operator independent system to delineate tumours in positron emission tomography scans. Comput Biol Med 2018;102:1-15.

27. Kijowski R, Liu F, Caliva F, et al. Deep learning for lesion detection, progression, and prediction of musculoskeletal disease. J Magn Reson Imaging 2020;52(6): 1607-19.

28. Cortes C, Jackel LD. Limits on learning machine accuracy imposed by data quality. Available at: https://www.aaai.org/Papers/KDD/1995/KDD95007.pdf. Accessed July 19, 2021.

29. Shinozaki A. Electronic medical records and machine learning in approaches to drug development. In: Cassidy JW, Taylor B, editors. Artificial intelligence in Oncology drug discovery and development. IntechOpen; 2020. https://doi.org/10.5772/ intechopen.92613.

30. Sachdeva S, Bhalla S. Semantic interoperability in standardized electronic health record databases. J Data Inf Qual 2012;3(1):1-37.

31. Martinez-Martin N, Magnus D. Privacy and ethical challenges in next-generation sequencing. Expert Rev Precis Med Drug Dev 2019;4(2):95-104.

32. Char DS, Lázaro-Muñoz G, Barnes A, et al. Genomic contraindications for heart transplantation. Pediatrics 2017;139(4). https://doi.org/10. 1542/peds.2016-3471.

33. A guide to disability rights laws. Available at: https://www.ada.gov/cguide.htm. Accessed July 24, 2021.

34. Genetic discrimination. Available at: https://www. genome.gov/about-genomics/policy-issues/GeneticDiscrimination. Accessed September 18, 2021.

35. Wall A, Lee GH, Maldonado J, et al. Genetic disease and intellectual disability as contraindications to transplant listing in the United States: a survey of heart, kidney, liver, and lung transplant programs. Pediatr Transpl 2020;24(7):e13837.

36. Lee SS-J, Fullerton SM, Saperstein A, et al. Ethics of inclusion: cultivate trust in precision medicine. Science 2019;364(6444):941-2.

37. Kuhlman C, Jackson L, Chunara R. No computation without representation: avoiding data and algorithm biases through diversity. Available at: http://arxiv. org/abs/2002.11836. Accessed September 10, 2021.

38. Burke W, Edwards KA, Goering S, et al. Achieving justice in genomic translation: re-thinking the pathway to benefit. Oxford University Press; 2011. Available at: https://play.google.com/store/books/ details?id=KA5pAgAAQBAJ.

39. Lewis ACF. Where bioethics meets machine ethics. Am J Bioeth 2020;20(11):22-4.

40. Burgart AM, Magnus D, Tabor HK, et al. Ethical challenges confronted when providing nusinersen treatment for spinal muscular atrophy. JAMA Pediatr 2018;172(2):188-92.

41. Chiruvella V, Guddati AK. Ethical issues in patient data ownership. Interact J Med Res 2021;10(2): e22269.

42. Milner LC, Garrison NA, Cho MK, etal. Genomics in the clinic: ethical and policy challenges in clinical next- 
generation sequencing programs at early adopter USA institutions. Per Med 2015;12(3):269-82.

43. Cutillo CM, Sharma KR, Foschini L, et al. Machine intelligence in healthcare-perspectives on trustworthiness, explainability, usability, and transparency. NPJ Digit Med 2020;3:47.

44. Pesapane F, Volonté C, Codari M, et al. Artificial intelligence as a medical device in radiology: ethical and regulatory issues in Europe and the United States. Insights Imaging 2018;9(5):745-53.

45. Institute of Medicine. Board on health sciences policy, committee on Accelerating rare diseases research and orphan Product development. Rare diseases and orphan Products: Accelerating research and development. National Academies Press; 2011. Available at: https://play.google.com/ store/books/details?id=HnYyeNIY3WwC.

46. Groft SC, Posada M, Taruscio D. Progress, challenges and global approaches to rare diseases. Acta Paediatr 2021;9. https://doi.org/10.1111/apa.15974.

47. Valdez R, Ouyang L, Bolen J. Public health and rare diseases: Oxymoron No more. Prev Chronic Dis 2016;13:E05.

48. Allen B, Dreyer K. The role of the ACR data science Institute in advancing health equity in radiology. J Am Coll Radiol 2019;16(4 Pt B):644-8.

49. Zou J, Schiebinger L. Ensuring that biomedical Al benefits diverse populations. EBioMedicine 2021; 67:103358.

50. Fletcher RR, Nakeshimana A, Olubeko O. Addressing fairness, bias, and appropriate use of artificial intelligence and machine learning in global health. Front Artif Intell 2020;3:561802.

51. Hurvitz N, Azmanov H, Kesler A, et al. Establishing a second-generation artificial intelligence-based system for improving diagnosis, treatment, and monitoring of patients with rare diseases. Eur J Hum Genet 2021; 19. https://doi.org/10.1038/s41431-021-00928-4.

52. Juth N. For the Sake of Justice: should We prioritize rare diseases? Health Care Anal 2017;25(1):1-20.

53. Scherman D. The dynamic and urgent path of rare disease and orphan drug research. Rare Dis Orphan Drugs J. Published online 2021. doi: 10.20517/rdodj.2021.01

54. Morris MA, Saboury B, Burkett B, et al. Reinventing radiology: big data and the future of medical imaging. J Thorac Imaging 2018;33(1):4-16.

55. Hosny A, Parmar C, Quackenbush J, et al. Artificial intelligence in radiology. Nat Rev Cancer 2018; 18(8):500-10.

56. Pirasteh A, Riedl C, Mayerhoefer ME, et al. PET/ MRI for neuroendocrine tumors: a match made in heaven or just another hype? Clin Transl Imaging 2019; 7(6):405-13.

57. Blumenrath SH, Lee BY, Low L, et al. Tackling rare diseases: clinical trials on chips. Exp Biol Med 2020;245(13):1155-62.
58. de Mello CPP, Rumsey J, Slaughter V, et al. A human-on-a-chip approach to tackling rare diseases. Drug Discov Today 2019;24(11):2139-51.

59. Paul A, Shen TC, Lee S, et al. Generalized Zeroshot Chest X-ray Diagnosis through Trait-Guided Multi-view Semantic Embedding with Self-training. IEEE Transactions on Medical Imaging. 2021.

60. Paul A, Tang YX, Shen TC,et al. Discriminative ensemble learning for few-shot chest $\mathrm{X}$-ray diagnosis. Medical Image Analysis. 2021 Feb 1;68: 101911.

61. Paul A, Tang YX, Summers RM. Fast few-shot transfer learning for disease identification from chest $x$-ray images using autoencoder ensemble. In Medical Imaging 2020: Computer-Aided Diagnosis 2020 Mar 16 (Vol. 11314, p. 1131407). International Society for Optics and Photonics.

62. Piñol M, Alves R, Teixidó I, et al. Rare disease discovery: an optimized disease ranking system. IEEE Trans Ind Inf 2017;13(3):1184-92.

63. Gurovich $Y$, Hanani $Y$, Bar $O$, et al. Identifying facial phenotypes of genetic disorders using deep learning. Nat Med 2019;25(1):60-4.

64. Ekins S, Puhl AC, Zorn KM, et al. Exploiting machine learning for end-to-end drug discovery and development. Nat Mater 2019;18(5):435-41.

65. Li Q, Zhao K, Bustamante CD, et al. Xrare: a machine learning method jointly modeling phenotypes and genetic evidence for rare disease diagnosis. Genet Med 2019;21(9):2126-34.

66. Progress in fighting rare diseases. Available at: https://www.phrma.org/en/Media/Progress-in-

Fighting-Rare-Diseases. Accessed July 19, 2021.

67. Gough A, Soto-Gutierrez A, Vernetti L, et al. Human biomimetic liver microphysiology systems in drug development and precision medicine. Nat Rev Gastroenterol Hepatol 2021;18(4):252-68.

68. Hargrove-Grimes P, Low LA, Tagle DA. Microphysiological systems: what it takes for community adoption. Exp Biol Med 2021. https://doi.org/10. 1177/15353702211008872.

69. Akobeng AK. Understanding diagnostic tests 2: likelihood ratios, pre- and post-test probabilities and their use in clinical practice. Acta Paediatr 2007;96(4):487-91.

70. Bond RR, Novotny T, Andrsova I, et al. Automation bias in medicine: the influence of automated diagnoses on interpreter accuracy and uncertainty when reading electrocardiograms. J Electrocardiol 2018; 51(6S):S6-11.

71. Rare disease advocacy - NORD's rare action NetworkTM - NORD (national organization for rare disorders). 2014. Available at: https://rarediseases.org/ for-patient-organizations/ways-partner/advocacy/.

Accessed July 18, 2021.

72. CME - NORD (National Organization for Rare Disorders). Available at: https://rarediseases.org/for- 
clinicians-and-researchers/resources/cme/. Accessed July 18, 2021.

73. Chan Zuckerberg. Initiative. Available at: https:// chanzuckerberg.com/. Accessed September 19, 2021.

74. Advocacy, Education \& Empowerment. Available at: http://www.geneticalliance.org/. Accessed July 21, 2021.

75. Schieppati A, Henter J-I, Daina E, et al. Why rare diseases are an important medical and social issue. Lancet 2008;371(9629):2039-41.

76. Genetic and Rare Diseases Information Center (GARD). Available at: https://ncats.nih.gov/gard. Accessed July 6, 2021.

77. Ali SR, Bryce J, Tan LE, et al. The EuRRECa Project as a model for data access and governance policies for rare disease registries that Collect clinical outcomes. Int J Environ Res Public Health 2020; 17(23). https://doi.org/10.3390/ijerph17238743.

78. Saboury B, Morris MA, Farhadi F, et al. Reinventing molecular imaging with total-body PET, Part I: technical revolution in Evolution. PET Clin 2020;15(4):427-38.

79. Prior F, Smith K, Sharma A, et al. The public cancer radiology imaging collections of the Cancer Imaging Archive. Sci Data 2017:4:170124.

80. El Emam K, Rodgers S, Malin B. Anonymising and sharing individual patient data. BMJ 2015;350: h1139. https://doi.org/10.1136/bmj.h1139.

81. Courbier S, Dimond R, Bros-Facer V. Share and protect our health data: an evidence based approach to rare disease patients' perspectives on data sharing and data protection - quantitative survey and recommendations. Orphanet J Rare Dis 2019;14(1):175.

82. Spencer K, Sanders C, Whitley EA, et al. Patient perspectives on sharing anonymized personal health data using a digital system for dynamic consent and research feedback: a Qualitative study. J Med Internet Res 2016;18(4):e66.

83. Figueiredo AS. Data sharing: convert challenges into opportunities. Front Public Health 2017;5:327.

84. Rare diseases. Available at: https://www.nih.gov/ about-nih/what-we-do/nih-turning-discovery-intohealth/rare-diseases. Accessed July 19, 2021.

85. Find Diseases We Study. Available at: https://www. rarediseasesnetwork.org/diseases. Accessed September 19, 2021.

86. Genetic Alliance - NORD (national organization for rare disorders). 2015. Available at: https:// rarediseases.org/organizations/genetic-alliance/. Accessed July 6, 2021.

87. Ghosh P (guha). The impact of data quality in the machine learning era - DATAVERSITY. Available at: https://www. dataversity.net/impact-data-qualitymachine-learning-era/. Accessed July 17, 2021.

88. Hallowell N, Parker M, Nellåker C. Big data phenotyping in rare diseases: some ethical issues. Genet Med 2019;21(2):272-4.
89. Yousefirizi F, Jha AK, Brosch-Lenz J, et al. Toward high-Throughput artificial intelligence-based segmentation in oncological PET imaging. PET Clin 2021;16(4):577-96.

90. Arslan M, Guzel M, Demirci M, et al. SMOTE and gaussian noise based sensor data augmentation. In: 2019 4th International Conference on computer science and Engineering (UBMK):Samsun, Turkey; 11-15 Sept. 2019:1-5. doi:10.1109/ UBMK.2019.8907003.

91. dos Santos Tanaka FHK, Aranha C. Data augmentation using GANs. Available at: http:// arxiv.org/abs/1904.09135.

92. Mikołajczyk A, Grochowski M. Data augmentation for improving deep learning in image classification problem. In: 2018 International Interdisciplinary PhD Workshop (IIPhDW):Cie, Poland. ieeexplore.ieee.org; 9-12 May 2018:117-122. doi:10.1109/ IIPHDW.2018.8388338

93. Frid-Adar M, Diamant I, Klang E, et al. GAN-based synthetic medical image augmentation for increased CNN performance in liver lesion classification. Neurocomputing 2018;321:321-31.

94. Frid-Adar M, Klang E, Amitai M, Goldberger J, Greenspan H. Synthetic data augmentation using GAN for improved liver lesion classification. In: 2018 IEEE 15th International Symposium on Biomedical Imaging:Washington, DC (ISBI 2018).

; 4-7 April 2018:289-293. doi:10.1109/
ISBI.2018.8363576

95. Zheng X, Chalasani T, Ghosal K, Lutz S, Smolic A. STaDA: Style Transfer as Data Augmentation. In: Proceedings of the 14th International Joint Conference on Computer Vision, Imaging and Computer Graphics Theory and Applications:Prague, Czech Republic. SCITEPRESS - Science and Technology Publications; 25-27, 2019. doi: 10.5220/0007353401070114

96. Qin R, Qiao K, Wang L, Zeng L, Chen J, Yan B. Weighted Focal Loss: An Effective Loss Function to Overcome Unbalance Problem of Chest X-ray 14. IOP Conference Series: Materials Science and Engineering:Chengdu, China. 19-22 July 2018;428:012022. doi:10.1088/1757-899x/428/1/ 012022

97. Zhi-huan ZE-HP. Cost sensitive support vector machines. Control Decis. 2006. Available at: https://en. cnki.com.cn/article_en/cjfdtotal-kzyc200604024. htm. Accessed September 10, 2021.

98. Wei Q, Ren Y, Hou R, et al. Anomaly detection for medical images based on a one-class classification. Medical imaging 2018: Computer-Aided diagnosis, 10575. SPIE; 2018. p. 375-80. https://doi. org/10.1117/12.2293408.

99. Ganapathy K. Artificial intelligence and healthcare regulatory and legal concerns. TMT 2021. https:// doi.org/10.30953/tmt.v6.252. 
100. Khosla N, Valdez R. A compilation of national plans, policies and government actions for rare diseases in 23 countries. Intractable Rare Dis Res 2018;7(4):213-22.

101. Hudson KL, Holohan MK, Collins FS. Keeping pace with the times-the genetic information Nondiscrimination act of 2008. N Engl J Med 2008;358(25): 2661-3.

102. Melnikova I. Rare diseases and orphan drugs. Nat Rev Drug Discov 2012;11(4):267-8.

103. Heemstra HE, van Weely S, Büller HA, et al. Translation of rare disease research into orphan drug development: disease matters. Drug Discov Today 2009;14(23-24):1166-73.

104. Liu B, He H, Luo H, et al. Artificial intelligence and big data facilitated targeted drug discovery. Stroke Vasc Neurol 2019;4(4):206-13.

105. New study shows the rate of drug approvals lower than previously reported. Available at: https:// archive.bio.org/media/press-release/new-studyshows-rate-drug-approvals-lower-previouslyreported. Accessed September 20, 2021.

106. Wang Y, Li E, Cherry SR, et al. Total-body PET kinetic modeling and potential opportunities using deep learning. PET Clin 2021;16(4):613-25.

107. Ataeinia B, Heidari P. Artificial intelligence and the future of diagnostic and therapeutic radiopharmaceutical development. PET Clin 2021;16(4):513-23.

108. Saboury B, Morris MA, Nikpanah M, et al. Reinventing molecular imaging with total-body PET, Part II. PET Clin 2020;15(4):463-75.

109. Morgenstern J. Why pretest probability is absolutely essential. First10EM blog. Published online 2019. doi:10.51684/firs.9601.

110. Nikpanah M, Saboury B, Farhadi F, et al. Pictorial review of the 18F-FDG PET/CT Manifestations of Erdheim-Chester disease. J Nucl Med 2019; 60(supplement 1):1141. Available at: https://jnm. snmjournals.org/content/60/supplement_1/1141. short. Accessed September 18, 2021.

111. Cuthbertson DJ, Barriuso J, Lamarca A, et al. The impact of 68Gallium DOTA PET/CT in managing patients with sporadic and familial pancreatic neuroendocrine tumours. Front Endocrinol 2021;12:654975. https://doi.org/10.3389/fendo.2021.654975.

112. Boér A, Szakáll S Jr, Klein I, et al. FDG PET imaging in hereditary thyroid cancer. Eur J Surg Oncol 2003;29(10):922-8.
113. July M, Santhanam P, Giovanella L, et al. Role of positron emission tomography imaging in Multiple Endocrine Neoplasia syndromes. Clin Physiol Funct Imaging 2018;38(1):4-9.

114. Duryea DM, Walker ER, Brian PL. Two foci of FDGavid secondary tumoral calcinosis incidentally noted in a patient with small-cell lung carcinoma after PET/CT. Radiol Case Rep 2014;9(4):998.

115. Oh J-R, Kulkarni H, Carreras C, et al. Ga-68 Somatostatin Receptor PET/CT in von Hippel-Lindau disease. Nucl Med Mol Imaging 2012;46(2): 129-33.

116. Hughes MS, Azoury SC, AssadipourY, etal. Prospective evaluation and treatment of familial carcinoid small intestine neuroendocrine tumors (SI-NETS). Surgery 2016;159(1):350-6.

117. Čtvrtlík F, Koranda P, Schovánek J, et al. Current diagnostic imaging of pheochromocytomas and implications for therapeutic strategy. Exp Ther Med 2018;15(4):3151-60.

118. Anand SS, Singh H, Dash AK. Clinical applications of PET and PET-CT. Armed Forces Med J India 2009;65(4):353-8.

119. Weisman AJ, Kim J, Lee I, et al. Automated quantification of baseline imaging PET metrics on FDG PET/CT images of pediatric Hodgkin lymphoma patients. EJNMMI Phys 2020;7(1):76.

120. Yang R, Yu Y. Artificial Convolutional neural Network in Object detection and semantic segmentation for medical imaging Analysis. Front Oncol 2021;11:638182. https://doi.org/10.3389/fonc. 2021.638182.

121. Lee Y-S, Krishnan A, Oughtred R, et al. A Computational Framework for Genome-wide Characterization of the Human Disease Landscape. Cell Syst. 2019;8(2):152-162.e6.

122. Saboury B, Morris MA, Nikpanah M, et al. Reinventing Molecular Imaging with Total-Body PET, Part II: Clinical Applications [published correction appears in PET Clin. 2021 Jan;16(1):xv]. PET Clin 2021;15(4):463-75. https://doi.org/10.1016/j.cpet. 2020.06.013.

123. Saboury B, Morris MA, Farhadi F, et al. Reinventing Molecular Imaging with Total-Body PET, Part I: Technical Revolution in Evolution. PET Clin 2020 Oct;15(4):427-38. https://doi.org/10.1016/j.cpet. 2020.06.012. 